\title{
Ultra B2 - Promoção de ligações covalentes do colágeno corneal (Corneal cross-linking') no tratamento de ceratocone: resultados preliminares
}

\author{
Corneal Cross-linking for the treatment of keratoconus: preliminary results
}

\author{
Mirko R. Jankov II ${ }^{1}$ \\ Farhad Hafezi $^{2}$ \\ Maja Beko ${ }^{3}$ \\ Zora Ignjatovic ${ }^{4}$ \\ Branislav Djurovic $^{5}$ \\ Vujica Markovic ${ }^{6}$ \\ PauloSchor $^{7}$
}

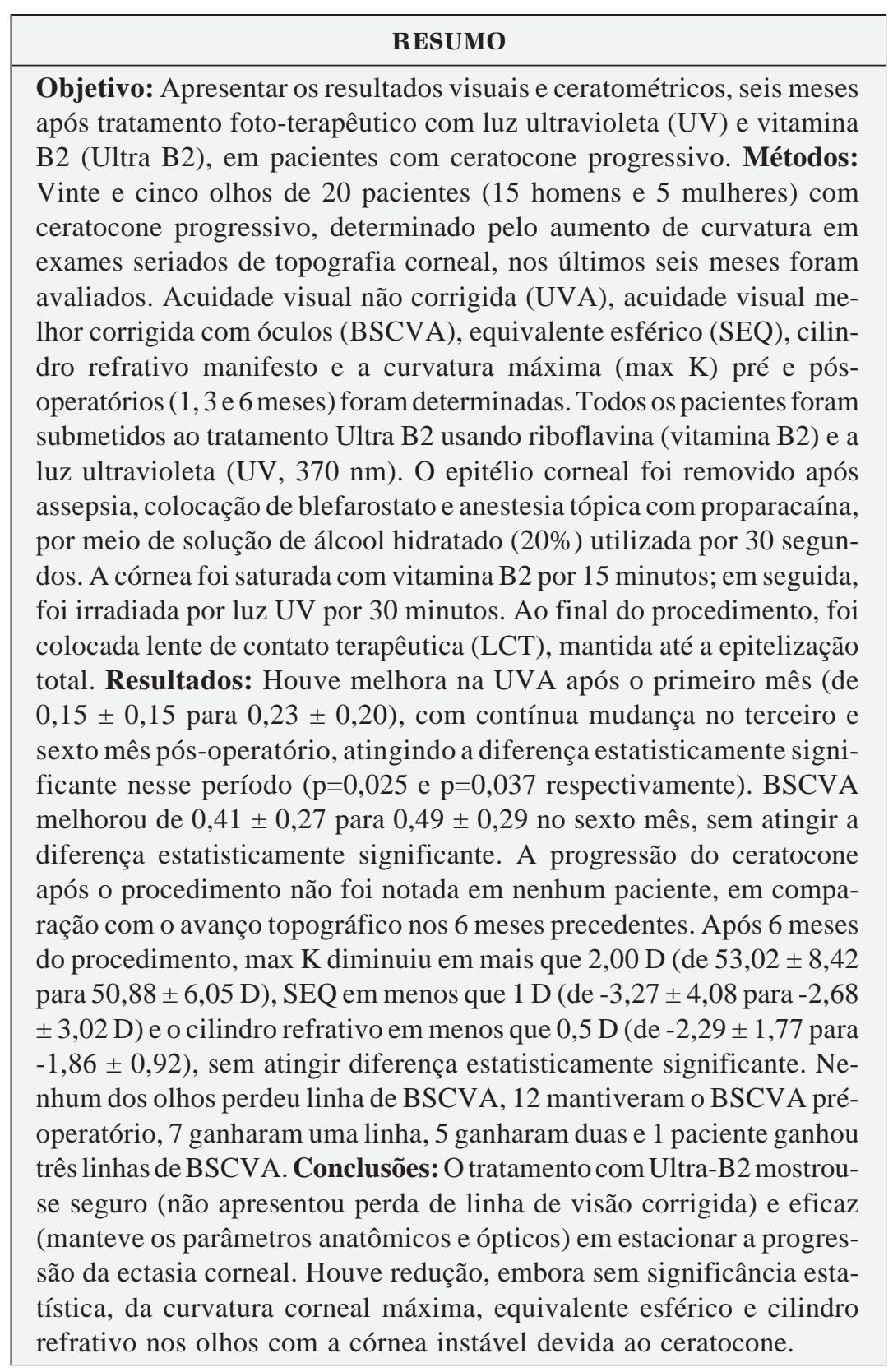

Descritores: Ceratocone/terapia; Colágeno/efeitos de radiação; Riboflavina/uso terapêutico; Terapia ultravioleta; Fototerapia; Raios ultravioleta; Reagentes para ligações cruzadas
Trabalho realizado no Milos Eye Hospital, Medical Academy - US Medical School, Belgrado, Sérvia. ${ }^{1}$ M.D. PhD, Laser Focus, Belgrado, Sérvia.

${ }^{2}$ M.D. PhD IROC - Institute of Refractive and Ophthalmic Surgery, Zurique, Suíça.

${ }^{3}$ M.D., Milos Eye Hospital, Medical Academy - US Medical School, Belgrado, Sérvia.

${ }^{4}$ M.D. MS, Milos Eye Hospital, Medical Academy - US Medical School, Belgrado, Sérvia.

${ }^{5}$ M.D. PhD, Milos Eye Hospital, Medical Academy - US Medical School, Belgrado, Sérvia.

${ }^{6}$ M.D. MS, Ocna Klinika, Medicinski Fakultet Univerziteta u Beogradu, Belgrado, Sérvia.

${ }^{7}$ M.D. PhD, Universidade Federal de São Paulo, UNIFESP - São Paulo (SP) - Brasil.

Endereço para correspondência: Mirko R. Jankov II. Cara Nikolaja II, 25/11000 Belgrado - Sérvia

E-mail: visioncare@mac.com

Recebido para publicação em 02.09.2007

Última versão recebida em 28.10.2008

Aprovação em 28.10.2008 


\section{INTRODUC̄̃̃O}

O ceratocone é uma doença bilateral assimétrica progressiva, na qual ocorre ectasia gradual da córnea devido à sua instabilidade biomecânica. Sua frequiência é de aproximadamente 1 em 2.000 na população geral, sem predileção pelo sexo $^{(1)}$. Usualmente a doença começa na puberdade e progride de maneira não constante, gradualmente tornando a superfície anterior da córnea cada vez mais irregular. O uso de óculos pode deixar de ser uma solução viável devido à irregularidade da córnea. Apesar da maioria dos pacientes ainda poder usar lente de contato rígida, em aproximadamente $20 \%$ dos casos a progressão é tal que a irregularidade da córnea leva a uma deterioração visual significativa, necessitando de transplante penetrante (TP) de córnea.

Portanto, uma vez que o paciente se torna intolerante ao uso de lentes de contato, as possibilidades de correção do ceratocone alternativas ao TP são escassas, de expectativas limitadas e resultados imprevisíveis do ponto de vista tanto anatômico quanto funcional ${ }^{(1)}$.

Nos últimos anos, o avanço das técnicas cirúrgicas dos transplantes lamelares ("deep anterior lamellar keratoplasty" - DALK e "intra-lamellar keratoplasty" - ILK), bem como a introdução dos anéis intra-corneais INTACS (Addition, Fremont, CA), e Ferrara (Ferrara Ophthalmics, Validolid, Spain) ou Keraring (Mediphacos Ophthalmic Professionals, São Paulo, Brazil) oferecem ao cirurgião possibilidades no manejo do ceratocone $e^{(2-5)}$.

Esses métodos tendem a regularizar a superfície anterior da córnea, porém baseiam-se na hipótese de que o estroma se manterá estável após o tratamento. Nos casos de astigmatismo irregular progressivo, como no ceratocone, degeneração marginal pelúcida ou ectasia iatrogênica após os tratamentos foto-refrativos, o estroma corneal encontra-se estruturalmente enfraquecido, havendo assim uma grande probabilidade de deterioração do quadro clínico com o passar do tempo. Seria necessário, portanto, um outro tratamento com a finalidade primária de estabilizar a córnea do ponto de vista biomecânico. Uma das propostas atualmente apresentadas é a promoção de ligações covalentes do colágeno do estroma corneal, mesmo antes de qualquer tentativa de modificação de sua superfície.

A promoção de ligações covalentes ("Cross-linking") do colágeno corneal (Ultra B2) é uma nova técnica de fortalecimento da córnea similar à foto-polimerização ${ }^{(6)}$. A combinação de riboflavina (vitamina B2), no papel de substância fotosensível, com a luz ultravioleta (UVA com $370 \mathrm{~nm}$ de comprimento de onda) induz ligações covalentes intra e inter-fibrilares, por meio da oxidação foto-sensível; o que, por sua vez, torna a córnea biomecanicamente mais resistente e estável ${ }^{(6-8)}$.

Objetivo deste trabalho foi de apresentar os resultados visuais e ceratométricos, seis meses após tratamento de fototerapêutico com luz ultravioleta (UV) e vitamina B2 (Ultra B2), em pacientes com ceratocone progressivo.

\section{MÉTODOS}

Vinte e cinco olhos de vinte pacientes ( 15 homens e 5 mulheres), sintomáticos, com a média de idade de $28 \pm 7$ (19 - 46) anos, foram incluídos neste estudo prospectivo. Os critérios de inclusão foram: ceratocone grau I a III, prova de progressão topográfica da doença nos últimos seis meses, e espessura corneal mínima de $400 \mu \mathrm{m}$. Os critérios de exclusão foram: ceratocone grau IV, ceratocone agudo com hidrópsia, opacidades corneais significativas, ceratite herpética, doenças vasculares de colágeno, auto-imunes ou outras doenças sistêmicas. O acompanhamento médio foi de 61 (4 a 7) meses. Todos os pacientes assinaram consentimento livre e esclarecido. $\mathrm{O}$ estudo foi aprovado pelo comitê de ética sob protocolo número 2006/0508.

A acuidade visual não corrigida (UVA) pré-operatória foi $0,15 \pm 0,15(0,01$ a 0,60$)$, a acuidade visual melhor corrigida com óculos (BSCVA) foi $0,41 \pm 0,27(0,05$ a 1,00$)$, o equivalente esférico (SEQ) foi $-3,27 \pm 4,08 \mathrm{D}(-13,50 \mathrm{a}+2,50)$, o cilindro manifesto refrativo foi $-2,29 \pm 1,77 \mathrm{D}(-6,00$ a $-0,25)$, enquanto a curvatura máxima (max $\mathrm{K}$ ) foi 53,02 \pm 8,42 D $(43,00$ a 67,20) (Tabela 1$)$.

\begin{tabular}{|c|c|c|c|c|}
\hline & Pré-operatório & 1 mês & 3 meses & 6 meses \\
\hline Cilindro [D] & 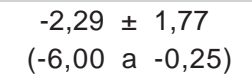 & $\begin{array}{c}-1,79 \quad \pm 1,36 \\
(-5,00 \text { a } 0,00)\end{array}$ & $\begin{array}{c}-1,84 \quad \pm 0,57 \\
(-2,75 \text { a }-1,25)\end{array}$ & $\begin{array}{c}-1,86 \pm 0,92 \\
(-3,00 \text { a } 0,00)\end{array}$ \\
\hline SEQ [D] & $\begin{array}{c}-3,27 \pm 4,08 \\
(-13,50 \text { a }+2,50)\end{array}$ & $\begin{array}{cc}-2,46 & \pm 2,92 \\
(-10,50 & a+1,75)\end{array}$ & $\begin{array}{c}-2,83 \pm 2,57 \\
(-6,13 \text { a }+1,13)\end{array}$ & $\begin{array}{c}-2,68 \pm 3,02 \\
(-8,00 a+1,50)\end{array}$ \\
\hline UVA & $\begin{array}{c}0,15 \pm 0,15 \\
(0,01 \text { a } 0,60)\end{array}$ & $\begin{array}{c}0,23 \pm 0,20 \\
(0,01 \text { a } 0,60)\end{array}$ & $\begin{array}{c}0,24 \text { 出 } 0,24 \\
(0,01 \text { a } 0,60) \text { * }\end{array}$ & $\begin{array}{c}0,26 \pm 0,25 \\
(0,01 \text { to } 0,60) \text { * }\end{array}$ \\
\hline BSCVA & $\begin{array}{c}0,41 \pm 0,27 \\
(0,05 \text { a } 1,00)\end{array}$ & $\begin{array}{r}0,49 \quad \pm \quad 0,25 \\
(0,20 \text { a } 1,00)\end{array}$ & $\begin{array}{c}0,46 \pm 0,29 \\
(0,10 \text { a } 1,00)\end{array}$ & $\begin{array}{c}0,49 \pm 0,29 \\
(0,10 \text { a } 1,00)\end{array}$ \\
\hline Max K & $\begin{array}{c}53,02 \quad \pm 8,42 \\
(43,00 \text { a } 67,20)\end{array}$ & $\begin{aligned} 51,18 & \pm 7,68 \\
(44,50 & \text { a } 65,70)\end{aligned}$ & $\begin{array}{c}49,73 \pm 6,24 \\
(44,40 \text { a } 60,40)\end{array}$ & $\begin{aligned} 50,88 & \pm 6,05 \\
(45,50 & \text { a } 60,40)\end{aligned}$ \\
\hline
\end{tabular}


$\mathrm{O}$ tratamento Ultra B2 foi feito pelo mesmo cirurgião (MJ) entre setembro de 2006 e março de 2007 em condições estéreis de centro cirúrgico. Colírio de anestésico tópico de proparacaína (Anestalcon, Alcon, Ft. Worth) foi aplicado e o epitélio corneal removido parcialmente em faixas da área central de $7 \mathrm{~mm}$ usando-se a espátula romba estéril. A solução de riboflavina a $0,1 \%$ (diluída em dextrano T-500 a 20\%) foi aplicada sob a forma de gotas, por 15 minutos antes, e durante todo o período de irradiação, a cada 2 a 3 minutos. A presença de coloração amarelada na câmara anterior, ao exame na lâmpada de fenda, foi confirmada antes do começo da irradiação pela luz ultravioleta.

Após a constatação da presença da riboflavina na câmara anterior, deu-se início à irradiação usando-se a lâmpada de raios UVA (370 nm, Peschkemed, Huenenberg, Suíça) na distância de trabalho de $6 \mathrm{~cm}$, por 30 minutos, com a irradiação de $3 \mathrm{~mW} / \mathrm{cm}^{2}\left(\sim 5.4 \mathrm{~J} / \mathrm{cm}^{2}\right)$ (Figura 1). Após o tratamento, colírio de antibiótico (Tobrex, Alcon, Ft. Worth) foi aplicado e a lente de contato terapêutica foi colocada e mantida até a re-epitelização completa, que ocorreu normalmente entre o $3^{\circ}$ e $5^{\circ}$ dia. Após a remoção da lente de contato, o colírio de antibiótico foi descontinuado, e colírio de corticóide (Pred Fort, Alcon, Ft. Wort) introduzido 4 vezes ao dia por um mês, diminuindo nos próximos dois meses até a parada completa.

\section{RESULTADOS}

A UVA melhorou no primeiro mês, de $0,15 \pm 0,15$ para $0,23 \pm$ 0,20 e continuou melhorando no terceiro e sexto mês pós-operatório, atingindo a diferença estatisticamente significante nos últimos dois períodos ( $\mathrm{p}=0,025$ e $\mathrm{p}=0,037$ respectivamente). A BSCVA melhorou de $0,4 \pm 0,27$ para $0,49 \pm 0,29$ no sexto mês, acompanhando assim o mesmo padrão, porém sem atingir a diferença estatisticamente significante (Figura 2, Tabela 1).

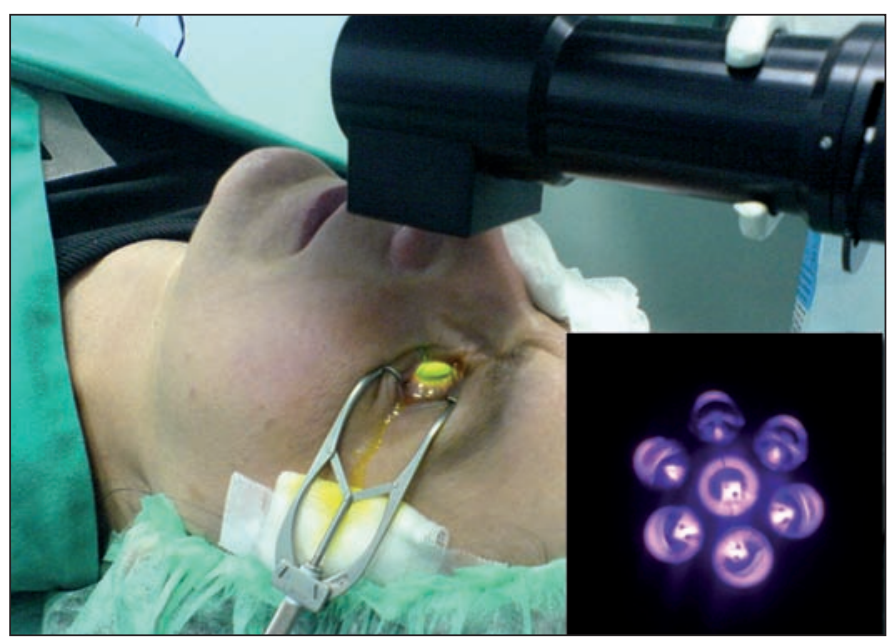

Figura 1 - Fotografia do tratamento Ultra B2 em progresso: nota-se a córnea embebida com riboflavina (amarelo) e irradiada pela luz UVA da lâmpada UVA. No canto inferior direito pode-se observar a lâmpada, na perspectiva do paciente.
A progressão do ceratocone não foi notada em nenhum dos pacientes, em comparação com o avanço topográfico que havia ocorrido em todos, nos 6 meses precedentes. Após 6 meses, max K diminuiu em mais que 2,00 D (de -53,02 $\pm 8,42$ D para 50,88 $\pm 6,05$ ) D, SEQ em menos que 1,00 D (de -3,27 \pm $4,08 \mathrm{D}$ para $-2,68 \pm 3,02 \mathrm{D}$ ) enquanto o cilindro refrativo também foi reduzido, em menos que $0,50 \mathrm{D}$ (de -2,29 $\pm 1,77 \mathrm{D}$ para $-1,86 \pm 0,92 \mathrm{D})$, sem atingir a diferença estatisticamente significante (Figuras 3 e 4, Tabela 1). Nenhum dos olhos perdeu linha de BSCVA; 12 mantiveram o BSCVA pré-operatório, 7 ganharam uma linha, 5 ganharam duas e 1 paciente ganhou três linhas de BSCVA.

\section{DISCUSSÃO}

A indicação principal para uso de Ultra B2 seria descontinuar o processo de ectasia nas doenças de córnea, tais como ceratocone e degeneração marginal pelúcida ${ }^{(6)}$. Ultra B2 pode ser utilizada também no tratamento, e talvez na profilaxia, das ectasias iatrogênicas após os tratamentos foto-refrativos que resultaram em um afinamento excessivo e progressivo da córnea ${ }^{(9)}$. Além das ectasias, esta técnica também pode ser utilizada para as condições de necrose ("melting") da córnea, como por exemplo nas ceratites infecciosas ${ }^{(10)}$.

O primeiro estudo prospectivo incluindo 23 olhos com ceratocone moderado ou avançado progressivo mostrou-se efetivo em parar a progressão do ceratocone no período de quatro $\operatorname{anos}^{(6)}$. Nesse estudo, a progressão média da ceratometria mais curva (max K) foi de 1,42 D em 52\% dos olhos, no período de seis meses anterior ao tratamento; enquanto após o tratamento, $70 \%$ dos olhos tinham uma redução de max $\mathrm{K}$ de 2,01 D em média. Além disso, o equivalente esférico (SEQ) diminuiu em média $1,14 \mathrm{D}$, enquanto $22 \%$ dos olhos-controle mostraram a progressão do ceratocone de $1,48 \mathrm{D}$. Os resultados do nosso estudo confirmaram os achados deste estudo inicial, reduzindo o curvatura máxima (max K) em 2,14 D e SEQ em 0,59 D.

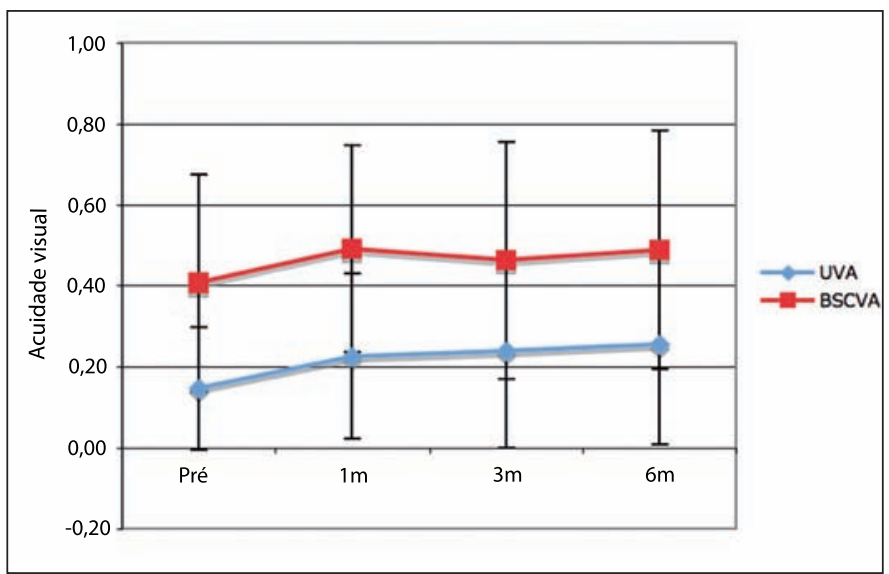

Figura 2 - Ultra B2 após $6 \mathrm{~m}$ - Melhoria da acuidade visual não corrigida (UVA) e melhor corrigida (BSCVA) 
Deve-se ressaltar que a finalidade primordial do tratamento Ultra B2 é de parar o avanço do ceratocone pelo enrijecimento da córnea e não de revertê-lo; caso a regressão ocorra, como os resultados de fato mostraram, esse é um efeito bem-vindo em decorrência do rearranjo das lamelas corneais e da matriz extracelular. Assim sendo, uma reação da córnea cada vez mais rígida, resultado de um número elevado de ligações covalentes, é esperada, produzindo uma redução da sua curvatura. Nestas circunstâncias, as lamelas mais rígidas têm um efeito biomecânico diferente em comparação ao seu comportamento anterior ao tratamento, e um novo balanço, resultando em um novo formato da córnea.

Levando-se em consideração a finalidade principal do tratamento Ultra B2 em parar o avanço do processo de ectasia, uma solução lógica seria de combinar dois métodos: por um lado, o tratamento que modificaria o formato de córnea (tais como anéis de Ferrara, INTACS ou mesmo PRK guiado pela topografia), e por outro o tratamento Ultra B2, que estabilizaria o efeito atingido por outro tratamento, desta maneira combinando o melhor das duas abordagens. Este estudo está em andamento, e será apresentado posteriormente.

Um exemplo ilustrativo é uma paciente feminina de 36 anos, usuária de lente de contato rígida gás-permeável por 18 anos, que procurou o nosso serviço pela instabilidade da lente de contato no olho esquerdo. Sua acuidade visual melhor cor-

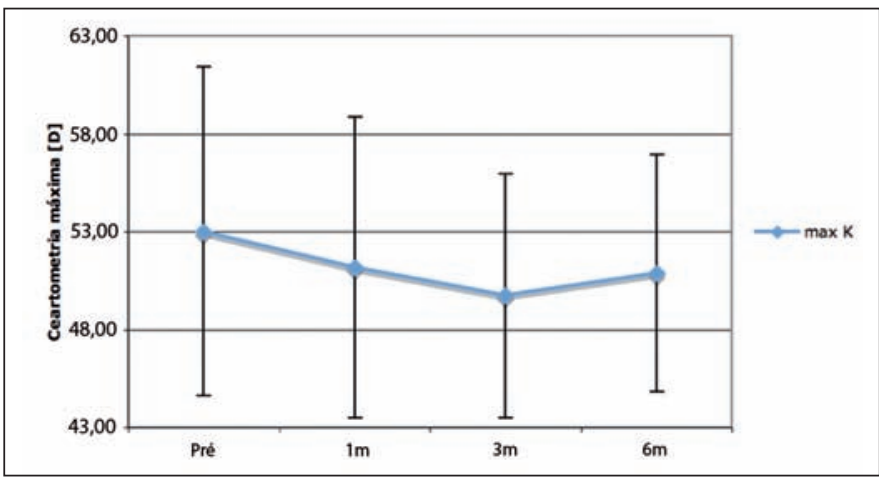

Figura 3 - Ultra B2 após 6 m - Diminuição da curvatura máxima corneal $(\max \mathrm{K})$

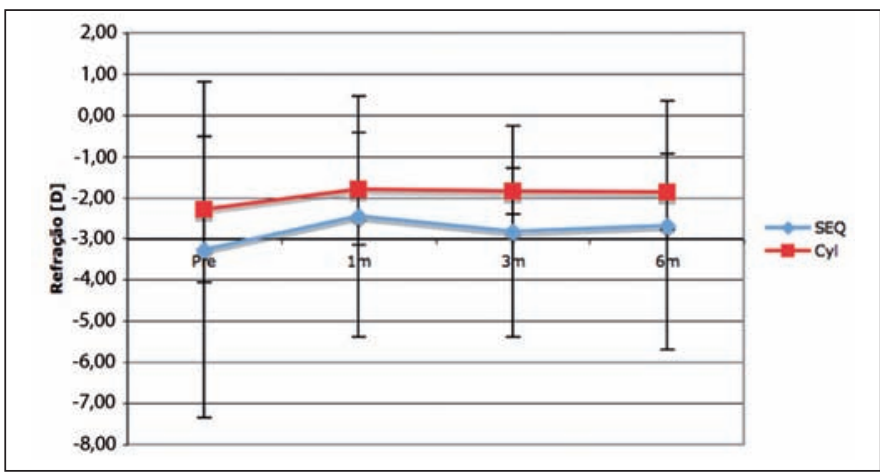

Figura 4 - Ultra B2 após $6 \mathrm{~m}$ - Evolução da refração em termos de equivalente esférico (SEQ) e cilindro manifesto (Cyl) rigida com a sua lente de contato foi 1,00, enquanto com óculos foi 20/50 com -2,50 DE/-1,50 DC x 90. Sua paquimetria foi de $484 \mu \mathrm{m}$, e após um mês sem uso de lente de contato, a topografia corneal mostrou o aspecto típico de ceratocone em ambos os olhos, pior no olho esquerdo. Esse olho foi tratado primeiramente, resultando em uma córnea mais regular e BSCVA de 0,62 com -3,50 DE / -1,50 DC x 90. Três meses após o tratamento Ultra B2, um PRK guiado pela topografia por meio do excimer laser WaveLight Allegretto WAVE e T-CAT software foi efetuado, melhorando a UVA da paciente para 0,80 e sua BSCVA a 1,00 parcial com -0,75 DE/-0,50 DC x 90, eliminando assim a necessidade de uso de lentes de contato (Figura 5).

Em seu estado natural, a córnea reduz a irradiação UVA em aproximadamente $30 \%$, enquanto o cristalino absorve aproximadamente $50 \%$ adicionais, sobrando aproximadamente $20 \%$ que passariam para a cavidade vítrea e a retina ${ }^{(11-12)}$. Por outro lado, com a presença da substância foto-sensibilizante riboflavina a 0,1\% impregnada no tecido corneal, $95 \%$ da irradiação é absorvido; portanto, a partir de uma irradiação de $3 \mathrm{~mW} / \mathrm{cm}^{2}$ na superfície corneal, somente $0,15 \mathrm{~mW} / \mathrm{cm}^{2}$ alcançam o endotélio ${ }^{(13)}$. Tais dados estão de acordo com os achados clínicos do nosso estudo, onde não detectamos anormalidades biomicroscópicas dignas de nota seis meses após a Ultra B2, tais como edema, falência endotelial ou alteração da transparência corneal.

Na porção anterior da córnea, a apoptose dos ceratócitos é seguida pela re-população dentro de um mês aproximadamente,

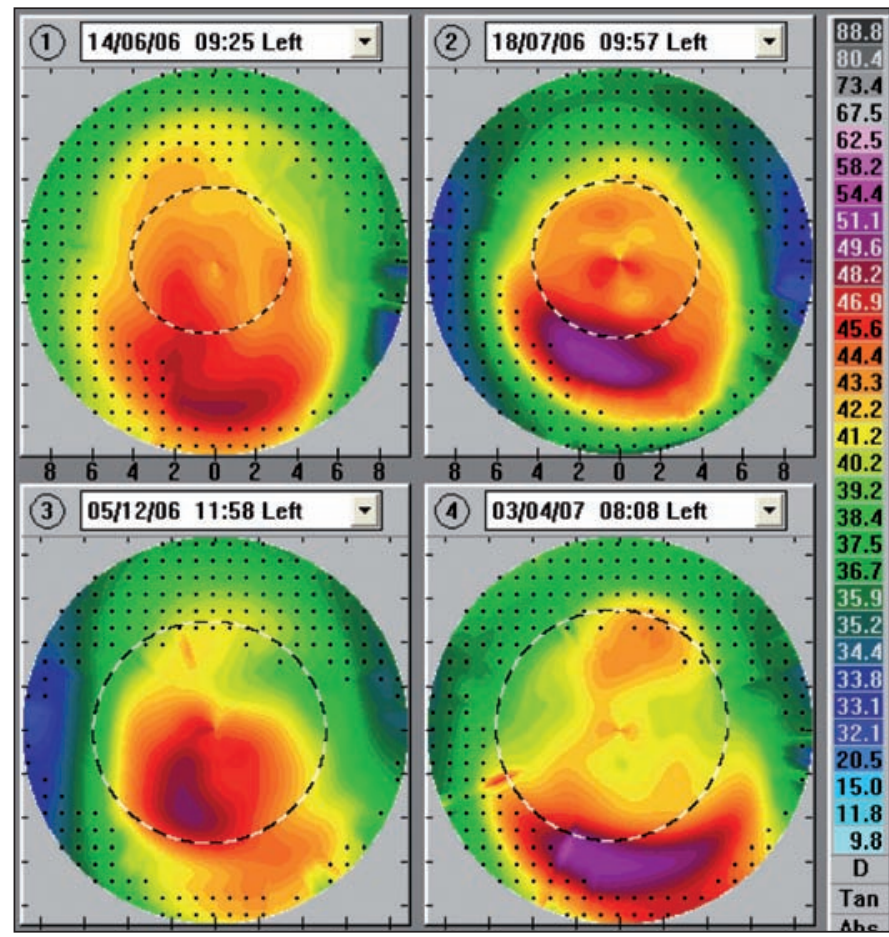

Figura 5 - Melhoria do formato corneal observada nos mapas topográficos tangenciais; 1 ) imediatamente após a remocão da lente de contato rígida gás-permeável (acima esquerda); 2) um mês após a remoção da lente de contato (acima direita); 3) cinco meses após o tratamento Ultra B2 (abaixo esquerda); 4) quatro meses após PRK guiada pela topografia usando-se T-CAT WaveLight Allegretto WAVE (abaixo direita) 
como pode ser visto com a microscopia confocal, voltando ao nível pré-operatório do ponto de vista morfológico dentro de seis meses após o tratamento ${ }^{(14)}$. O patamar da irradiação citotóxica para o endotélio após o tratamento combinado de riboflavina/ UVA é de $0,36 \mathrm{~mW} / \mathrm{cm}^{2}\left(\sim 0,65 \mathrm{~J} / \mathrm{cm}^{2}\right)$, o que ocorre nas córneas com as espessuras inferiores a $400 \mu \mathrm{m}$ no caso de irradiação padrão de $3 \mathrm{~mW} / \mathrm{cm}^{2}$ de irradiação $\left(\sim 5,4 \mathrm{~J} / \mathrm{cm}^{2}\right)$ na superfície corneal $^{(13)}$. Portanto, a medição detalhada da paquimetria corneal mínima (não necessariamente a central) é essencial e deve ser sempre obtida, preferencialmente usando-se o mapa paquimétrico com escaneamento por fendas ou tomografia corneal.

O tratamento Ultra B2 foi utilizado também para o controle do avanço da ectasia iatrogênica nos olhos após as ablações excessivas por excimer laser. Num estudo publicado na Alemanha, o estado biomecânico da córnea foi estabilizado com a parada da progressão topográfica e refrativa nos olhos após a ectasia iatrogênica no período de $18 \operatorname{meses}^{\left({ }^{(9)}\right.}$. A técnica cirúrgica foi igual à descrita para o tratamento de ceratocone, já que a riboflavina penetra livremente entre o retalho ("flap") e o leito estromal.

Uma outra área de aplicações de Ultra B2 foi a ulceração de córnea progressiva, onde o tratamento Ultra B2 conseguiu parar o processo de ceratólise nos olhos tratados ${ }^{(10)}$. Em três dos quatro pacientes com ulcerações de córnea progressivos e necrose de córnea, o Ultra B2 fez com que o processo se revertesse e a anatomia de córnea se recuperasse, já que Ultra B2 restabelece a resistência da córnea às enzimas colagenolíticas ${ }^{(15)}$, enquanto a irradiação UVA elimina os agentes infecciosos.

Até a presente data, não houve relatos de efeitos colaterais ou complicações nos estudos clínicos multicêntricos controlados de Ultra B2. O edema leve transitório estromal e epitelial é comum nos olhos tratados, assim como o aspecto levemente esfumaçado com a aparência de fios de algodão no estroma corneal, o que usualmente regride de 4 a 6 semanas após o tratamento (Figuras 6 e 7).

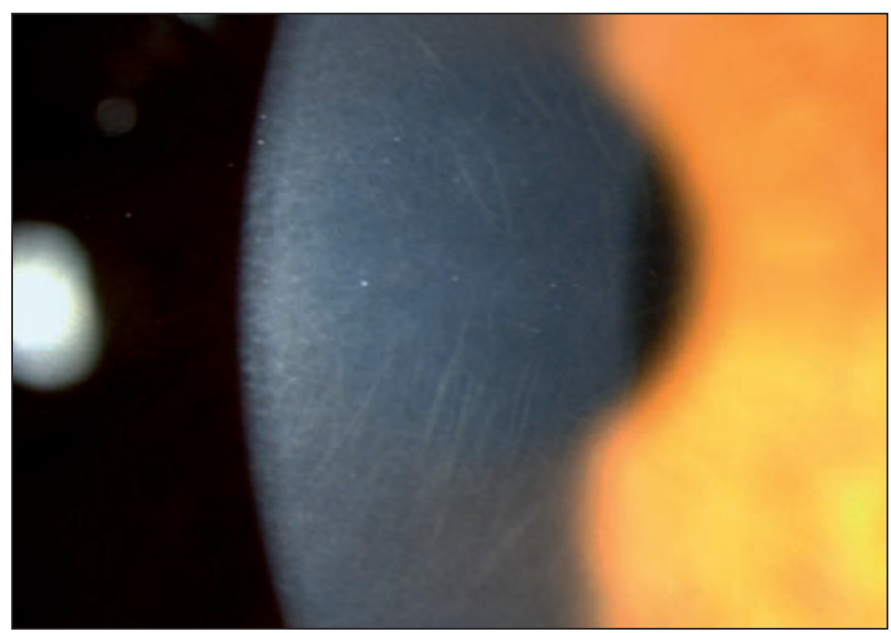

Figura 6 - Fotografia da biomicroscopia corneal um mês após o tratamento Ultra B2: discreto edema estromal e epitelial pode ser observado, junto com o aspecto levemente enfumaçado e a aparência de fios de algodão no estroma corneal. (Cortesia do Dr. E. Coskunseven, MD, Turquia).

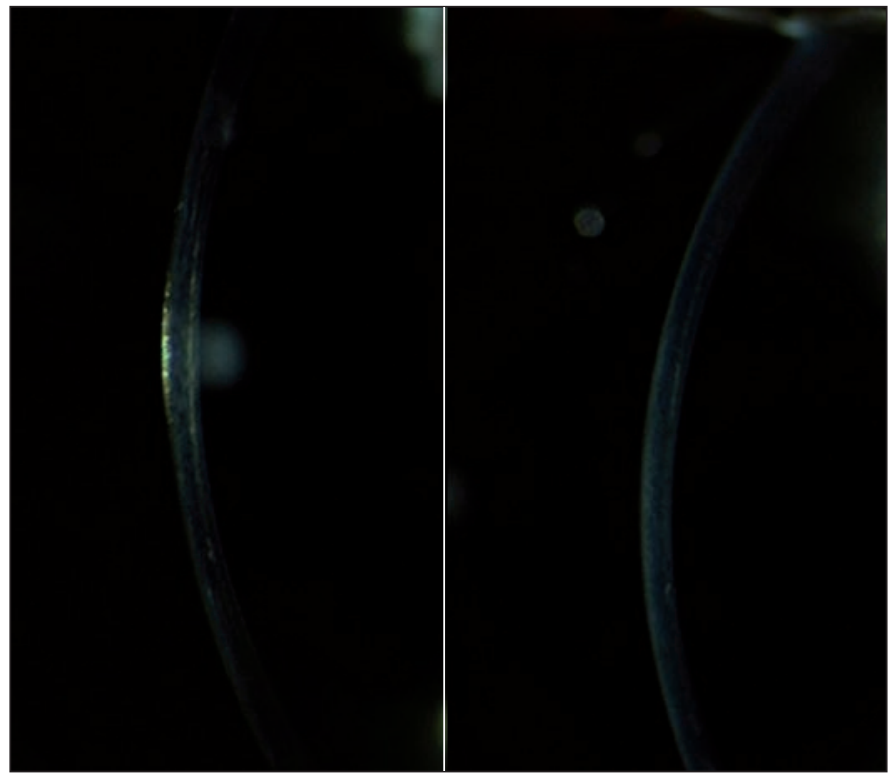

Figura 7 - Biomicroscopia corneal no terceiro dia, imediatamente após a retirada da lente de contato terapêutica. Nota-se uma linha pálida de demarcação entre a porção anterior da córnea com edema, e a porção normal mais profunda (esquerda); três meses após o tratamento Ultra B2 a linha desaparece quase por completo, acompanhando o retorno da anatomia e fisiologia funcional da córnea ao seu estado anterior ao tratamento (direita) (Cortesia do prof. T. Seiler, MD PhD. Suíça).

O tratamento Ultra B2 mostrou-se seguro (não apresentou perda de linha de visão corrigida) e eficaz (manteve os parâmetros anatômicos e ópticos) em estacionar a progressão da ectasia corneal. Houve redução, embora sem significância estatística, da curvatura corneal máxima, equivalente esférico e cilindro refrativo nos olhos com a córnea instável devida ao ceratocone.

\section{ABSTRACT}

Purpose: To present early visual and keratometric results for corneal cross-linking with riboflavin and UV irradiation in patients with progressive keratoconus. Methods: Twentyfive eyes of twenty patients ( 15 males and 5 females) with a progressive keratoconus in the previous 6 months were followed. Unaided visual acuity (UVA), best spectacle corrected visual acuity (BSCVA), spherical equivalent (SEQ), manifest cylinder, and maximal corneal curvature ( $\max \mathrm{K}$ ) values were followed at 1, 3 and 6 months. All patients were submitted to corneal cross-linking using riboflavin (vitamin B2) as the photosensitizer and ultraviolet light (UV, wavelength $370 \mathrm{~nm}$ ). Epithelium was removed with 20\% alcohol, cornea was soaked with vitamin B2 for $15 \mathrm{~min}$, and then irradiated with UV light for $30 \mathrm{~min}$, after which a bandage contact lens (BCL) was placed. Results: UVA increased after one month (from $0.15 \pm 0.15$ to $0.23 \pm 0.20$ ), and went on increasing at 3 and 6 months, reaching statistical significance $(\mathrm{p}=0.025 \mathrm{e}$ $\mathrm{p}=0.037$, respectively). BSCVA increased from $0.41 \pm 0.27$ to $0.49 \pm 0.29$ at month six, without reaching statistical signifi- 
cance at any time point. Progression of keratoconus stopped in all patients, in contrast with progression in all of them in the six-month period prior to the surgery. Max $\mathrm{K}$ decreased by more than $2 \mathrm{D}$ (from $53.02 \pm 8.42$ to $50.88 \pm 6.05 \mathrm{D}$ ), SEQ less that $1 \mathrm{D}$ (from $-3.27 \pm 4.08$ to $-2.68 \pm 3.02 \mathrm{D}$ ), while refractive cylinder decreased less than $0.5 \mathrm{D}$ (from $-2.29 \pm$ 1.77 to $-1.86 \pm 0.92 \mathrm{D})$, without reaching a statistically significant difference. None of the eyes lost any line of BSCVA, 12 maintained the preoperative BSCVA, 7 gained one line, 5 gained two lines, and 1 patient gained three lines of BSCVA. Conclusions: Corneal cross-linking with riboflavin and UV light seems to be a safe (no loss of BSCVA) and effective (anatomical and optical properties maintained) procedure, which has shown to stop the progression of the keratoconus: a reduction, although not statistically significant, of the corneal curvature, spherical equivalent and refractive cylinder took place in patients where previous progression of keratoconus had been described.

Keywords: Keratoconus/therapy; Collagen/radiation effects; Riboflavin/therapeutic use; Ultraviolet therapy; Phototherapy; Ultraviolet rays; Cross-linking reagents

\section{REFERÊNCIAS}

1. Lindstrom RL. The surgical correction of astigmatism: a clinician's perspective. Refract Corneal Surg. 1990;6(6):441-54.
2. Shimmura S, Tsubota K. Deep anterior lamellar keratoplasty. Curr Opin Ophthalmol. 2006;17(4):349-55.

3. Tan BU, Purcell TL, Torres LF, Schanzlin DJ. New surgical approaches to the management of keratoconus and post-LASIK ectasia. Trans Am Ophthalmol Soc. 2006;104(12):212-20.

4. Rabinowitz YS. Intacs for keratoconus. Curr Opin Ophthalmol. 2007;18(4):279-83.

5. Miranda D, Sartori M, Francesconi C, Allemann N, Ferrara P, Campos M. Ferrara intrastromal corneal ring segments for severe keratoconus. J Refract Surg. 2003;19(6):645-53.

6. Wollensak G, Spoerl E, Seiler T. Riboflavin/ultraviolet-a-induced collagen crosslinking for the treatment of keratoconus. Am J Ophthalmol. 2003;135(5):620-7.

7. Spoerl E, Huhle M, Seiler T. Induction of cross-links in corneal tissue. Exp Eye Res. 1998;66(1):97-103.

8. Spoerl E, Seiler T. Techniques for stiffening the cornea. J Refract Surg. 1999; 15(6):711-3.

9. Kohlhaas M, Spoerl E, Speck A, Schilde T, Sandner D, Pillunat LE. [A new treatment of keratectasia after LASIK by using collagen with riboflavin/UVA light cross-linking]. Klin Monatsbl Augenheilkd. 2005;222(5):430-6. German.

10. Schnitzler E, Spörl E, Seiler T. [Irradiation of cornea with ultraviolet light and riboflavin administration as a new treatment for erosive corneal processes, preliminary results in four patients]. Klin Monatsbl Augenheilkd. 2000;217(3):190-3. German.

11. Wollensak G, Spoerl E, Reber F, Seiler T. Keratocyte cytotoxicity of riboflavin/UVA-treatment in vitro. Eye. 2004;18(7):718-22.

12. Wollensak G, Spoerl E, Wilsch M, Seiler T. Keratocyte apoptosis after corneal collagen cross-linking using riboflavin/UVA treatment. Cornea. 2004;23(1):43-9.

13. Wollensak G, Spoerl E, Wilsch M, Seiler T. Endothelial cell damage after riboflavin-ultraviolet-A treatment in the rabbit. J Cataract Refract Surg. 2003; 29(9):1786-90.

14. Mazzotta C, Balestrazzi A, Traversi C, Baiocchi S, Caporossi T, Tommasi C, Caporossi A. Treatment of progressive keratoconus by riboflavin-UVA-induced cross-linking of corneal collagen: ultrastructural analysis by Heidelberg Retinal Tomograph II in vivo confocal microscopy in humans. Cornea. 2007; 26(4):390-7.

15. Spoerl E, Wollensak G, Seiler T. Increased resistance of crosslinked cornea against enzymatic digestion. Curr Eye Res. 2004;29(1):35-40. 